\title{
A multibody strategy for deformable grains
}

\author{
Guilhem Mollon ${ }^{1, *}$ \\ ${ }^{1}$ LaMCoS, INSA Lyon, UMR 5259, Université de Lyon, CNRS, 69621 Villeurbanne Cedex, France
}

\begin{abstract}
This article presents a numerical framework dedicated to the simulation of granular materials with highly deformable grains. This framework is based on a multibody meshfree strategy, which makes it possible to account for the constitutive model of the material composing each grain and for possibly complex contact laws (e.g. adhesion, friction, etc.). The main principles of the approach are first presented, and two illustrative cases are then detailed in order to emphasize its potential in several domains of the granular science.
\end{abstract}

\section{Introduction}

Discrete-Element modelling (DEM) has become a widely-used numerical method for fine and predictive modelling of granular materials in several industrial and scientific fields (e.g. geomechanics, food industry, chemistry, etc.). In this framework, each grain is considered as a rigid body which follows the laws of motion and interacts with other bodies by the means of contacts. In some situations, however, the assumption of rigid bodies enters into contradiction with the observed physical behaviours. This is the case when the material composing the grains is relatively soft (e.g. ductile powders), or when this material is submitted to very high loadings (e.g. tribology). To go beyond this limitation, this article presents a novel numerical framework which makes it possible to reproduce the compliance of the material at the grain scale. Two illustrative cases are then proposed in order to emphasize the potential of the approach.

\section{Numerical framework}

The numerical framework proposed hereafter is based on the following choices, some of which being detailed in [1]:

- The granular material is composed of a set of individual grains, and each grain is delimited by a closed boundary. The present approach is restricted to two dimensions.

- Each grain is a deformable body, and the displacement field is interpolated on its domain using a meshfree description based on a certain number of field nodes, with two degrees of freedom in displacement each (Fig. 1). The shape functions are based on Moving Least Square (MLS) interpolation, as first proposed in the Element-Free Galerkin framework [2] (Fig. 2).
- Contact forces are computed on the basis of a two-pass node-to-segment penalized formulation, hence considering the interaction between each field node located on the boundary of a given grain and the boundaries of the surrounding grains.

- At a given time step, a classical weak-form of the continuum mechanics equations is considered for the whole problem (like in Finite Element Method). Numerical integration is performed on each grain using a Gauss quadrature on a triangular mesh. An adaptive explicit scheme is used for time integration.

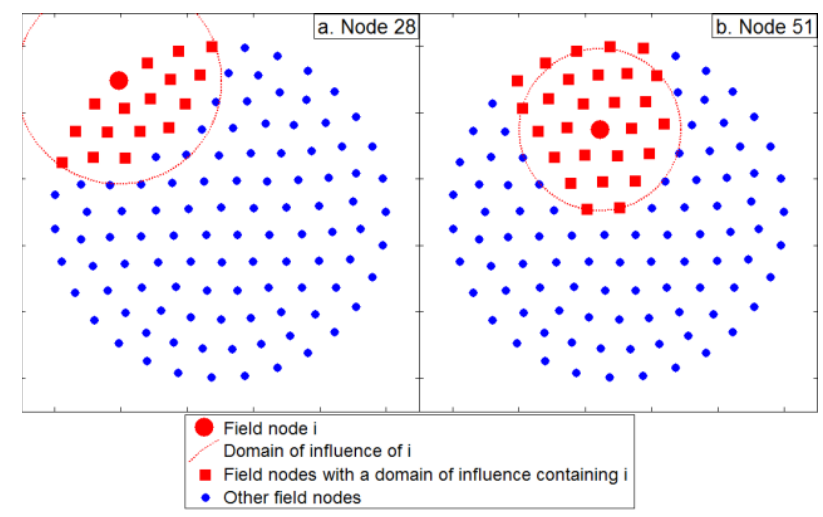

Fig. 1. Meshfree discretization of a circular grain, focusing on two particular nodes. a. node 28 (boundary node) ; b. node 51 (inner node).

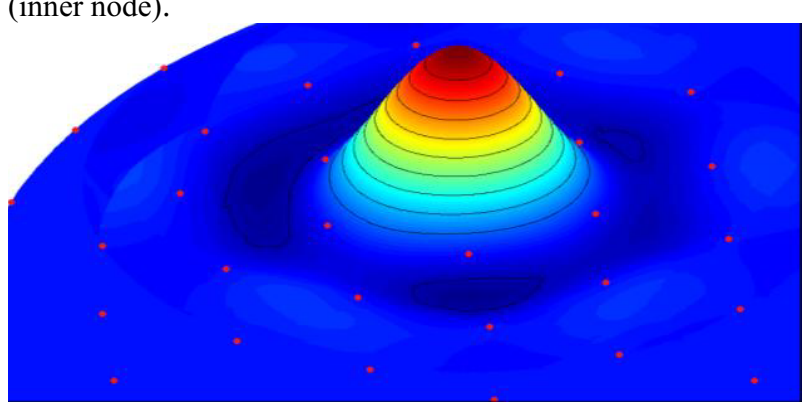

Fig. 2. MLS shape functions of node 51 . 


\section{First illustrative case - Tribology}

The third body concept is often used to describe the thin layer of degraded material which separates contacting bodies during sliding contact (Fig. 3). This third body may be either natural (i.e. produced by the degradation of the contacting surfaces and by matter entering into the contact) or artificial (i.e. placed there for a given purpose, e.g. grease). In any case, it is submitted to high loading (up to several $\mathrm{GPa}$ ) and strong shearing. If such phenomena as friction and wear are to be fully understood, an accurate modelling of the third body is hence crucial. In recent years, simulation methods based on DEM have been successful in understanding several properties of the third-body behavior [3]. The next stage, however, is to render each third body particle compliant in order to be able to deal with important phenomena such as complex constitutive laws, plasticity, Poisson effect, surface adhesion, etc.

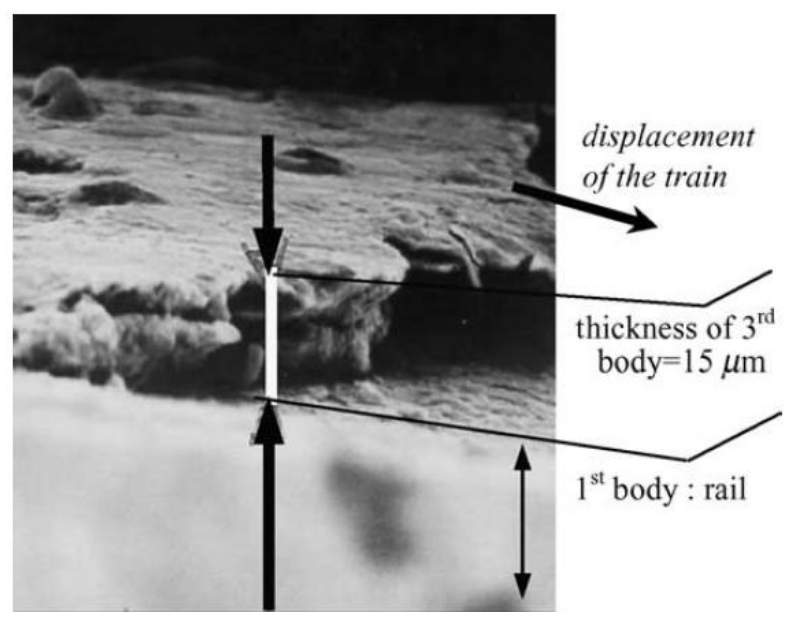

Fig. 3. Third body layer in a wheel-rail contact (from [4]).

To illustrate the potential use of multibody meshfree modelling in this framework, a simple study case is described in Fig. 4. In this typical tribological situation, two deformable bodies (so-called "first bodies") are located below and above a collection of 500 deformable discs which represent the third body. The lower boundary is fixed, the upper boundary is submitted to an imposed vertical displacement (in order to compress the third body) and to a horizontal velocity (in order to shear it continuously in the same way as in a sliding contact). Periodic boundaries are applied on each lateral side. An arbitrary roughness is introduced at the interfaces between first and third bodies.

The system is hence composed of 502 bodies discretized by roughly 75,000 field nodes, reaching a moderate size by modern computational standards (i.e. about 150,000 degrees of freedom). First bodies and third body particles follow an hyperelastic constitutive model, and the contact law is adhesive in order to reflect the typical behaviour of solid interfaces at sub-micrometric scale. In the case presented here, low third body stiffness and moderate contact adhesion are considered.

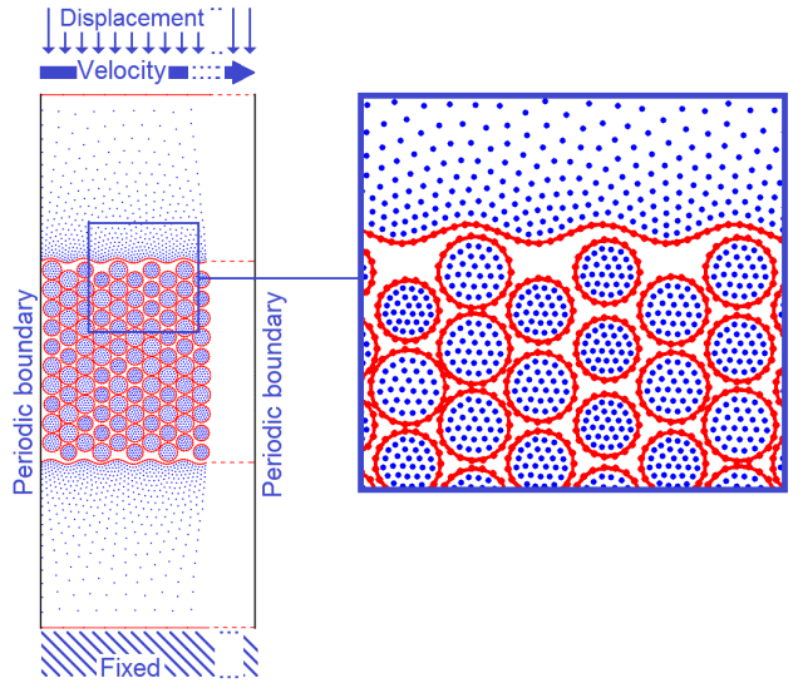

Fig. 4. Layout of the first illustrative case related to tribology.

Some numerical results are presented in Fig. 5, which shows four successive stages of the simulation. Situation (a) corresponds to the initial state of the system, (b) corresponds to the first stages of the loading, (c) to the installation of a flow regime in the third body, and (d) to the final stage. For these four instants, both stress and velocity fields are provided

These results show that, in the first stages of the loading, the stress field looks similar to the force network which is usually observed in hard granular materials (i.e. force chains mostly oriented in the direction of the main effort). However, when the loading is high enough to strongly deform the grains, this network evolves towards a continuous and complex stress pattern. The use of the same discretization method for the first and third bodies also makes it possible to observe the stress patterns in the first bodies, and to study their degradation on a quantitative basis.

The velocity fields show that, in this specific case, the velocity jump between the two first bodies is firstly accommodated by a rather smooth velocity gradient (i.e. bulk shearing in the third body), although Fig. 5c. shows that this gradient is actually controlled by localized slipping events. However, after a period of sliding, the accommodation regime evolves naturally towards a single slip line, with a moderate macroscopic roughness. Simultaneously to this change of regime, quantitative data (not provided here) show a reduction of the rate of energy dissipation in the system, i..e. a reduction of the apparent coefficient of friction. This kind of behaviour is consistent with some tribological observations, and could not be observed with rigid grains.

Other simulations with different parameters (stiffness of the third body, adhesion, loading, velocity, etc.) led to a large variety of local phenomena (bulk-shearing, wallslip, rollers, stick-slip, etc.), which reflect the varied phenomenology which is observed in experiments. 


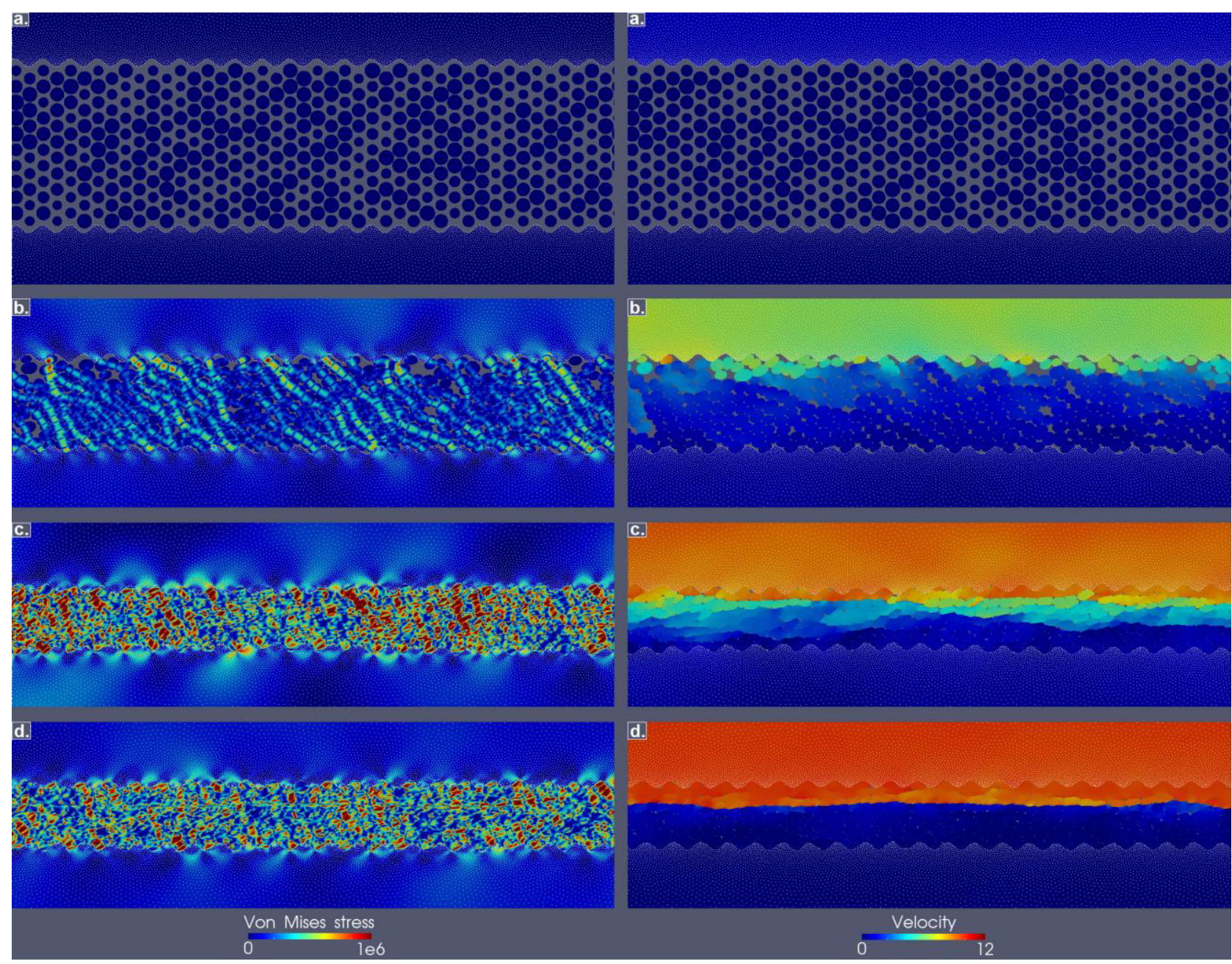

Fig. 5. Four snapshots of a tribological simulation. Left: Von-Mises stress field ; Right: Velocity norm field (arbitrary units).
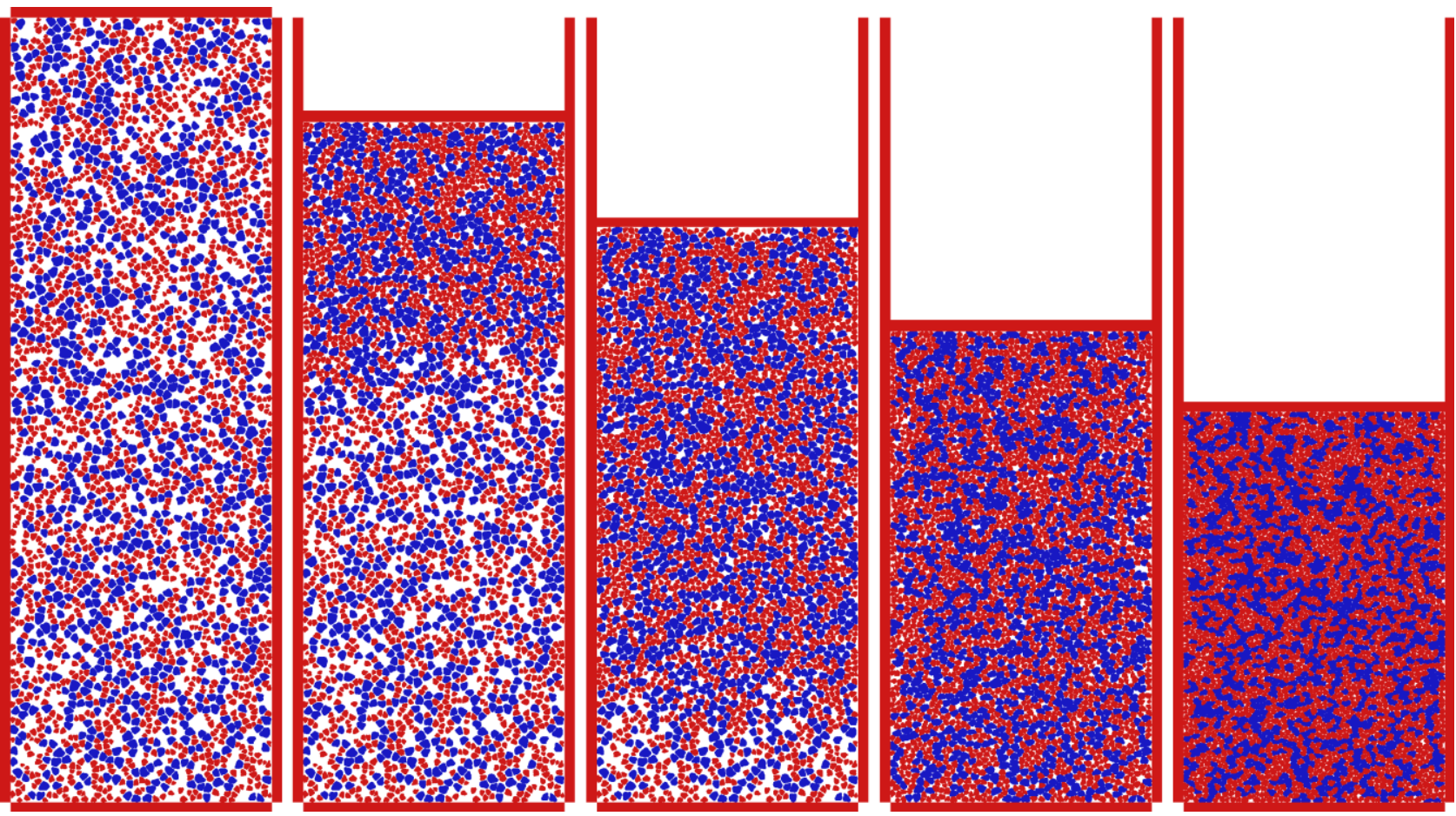

Fig. 6. Simulation of the compaction of a mixed powders with 3000 grains. In red: rigid bodies ; In blue: deformable bodies. 

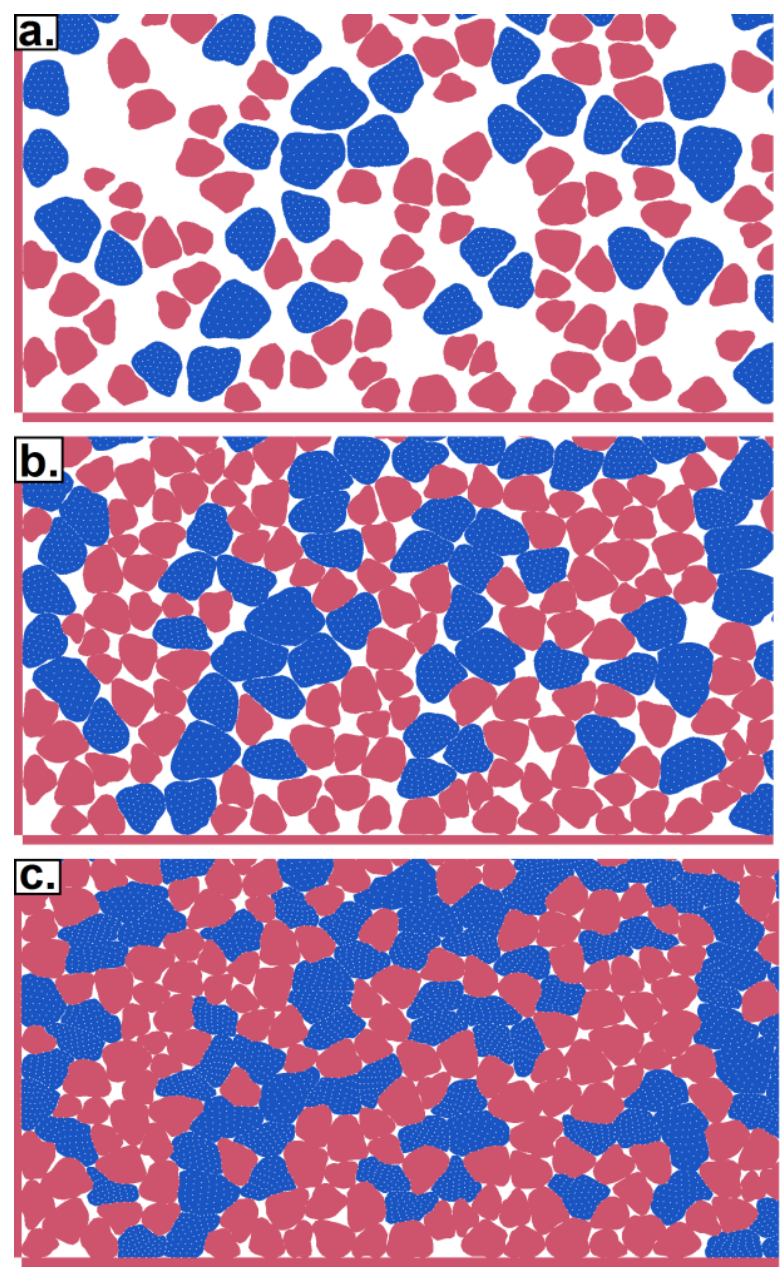

Fig. 7. Zoom on the lower-left corner of the simulation shown in Fig. 6. ; a. Initial state ; b. Beginning of powder compaction ; c. Final state.

\section{Second illustrative case - Mixed powders compaction}

The second illustrative case aims at showing the ability of the numerical framework to deal with rigid and deformable bodies within the same simulation. For this purpose, a powder compaction setup is simulated. The granular sample is generated with realistic shapes on a Fourier-Voronoi basis (see [5]). It is composed of 2000 rigid grains (as in a DEM simulation) and 1000 deformable grains (discretized using the meshfree technique presented above). The granular sample is positioned between four rigid walls, and the upper wall is submitted to an imposed downwards velocity (Fig. 6). This situation is typical of the compaction of a powder with two types of grains composed of materials with very different Young moduli.

The interactions between rigid and deformable grains is handled in a natural way in the code, since the contour of the rigid grains is discretized by a set of nodes and of segments: the same contact algorithm may then be applied for any couple of grains, either rigid or deformable. Coulomb friction with a coefficient of 0.5 is considered for all the contacts of this simulation.
Fig. 7 shows a zoomed view on the lower-left corner of the compacting box, at three different stages of the simulation. Stage (a) corresponds to the initial state, stage (b) corresponds to a compaction stage where the compliant grains are not yet strongly deformed, and stage (c) corresponds to the final state of the simulation. From this figure, it is evident that the numerical framework is able to capture the difference of behaviour between the two populations of grains: the final distribution of the voids is concentrated around the contacts between rigid grains, while deformable grains are able to "fill" the voids and to strongly reduce the local porosity.

\section{Conclusion and prospects}

A novel numerical framework dedicated to highly deformable granular materials was presented in this article. Two illustrative cases were provided in order to demonstrate the applicability of the code to a wide variety of situations where the compliance of the grains should be considered. The next stages of the work will be dedicated to a convergence study (in terms of time and space discretizations) and to experimental validations.

Future versions of the code will include several features which will enable to represent in a more accurate manner the local physics of highly loaded contacts with a third body, for tribological applications:

-Enriched behaviour of the grains (plasticity, viscosity, breakage, etc.) and of the first bodies (degradation, fatigue, etc.)

-Enriched contact laws based on physical models (e.g. JKR, Van der Waals, etc.)

-Heat creation and diffusion

-Fluid/grain coupling

A particular attention will also be paid to the computational efficiency of the code, in order to be able to use it in a High Performance Computing (HPC) framework.

\section{References}

1. G. Mollon, Int. J. Num. Meth. Eng., accepted (2016)

2. T. Belitchko, Y.Y. Lu, L. Gu, Int. J. Num. Meth. Eng. 37, 229-256 (1994)

3. M. Champagne, M. Renouf, Y. Berthier, ASME J. Tribol. 136(2), 021603 (2014)

4. S. Descartes, C. Desrayaud, E. Niccolibi, Y. Berthier, Wear 258, 1081-1090 (2005)

5. G. Mollon, J. Zhao, Gran. Mat. 14, 621-638 (2012) 